This item was submitted to Loughborough's Research Repository by the author.

Items in Figshare are protected by copyright, with all rights reserved, unless otherwise indicated.

\title{
A multiplexed electronic architecture for opto-electronic patch sensor to effectively monitor heart rate and oxygen saturation
}

PLEASE CITE THE PUBLISHED VERSION

https://doi.org/10.1117/12.2287833

\section{PUBLISHER}

(C) Society of Photo-Optical Instrumentation Engineers (SPIE)

\section{VERSION}

VoR (Version of Record)

\section{PUBLISHER STATEMENT}

This work is made available according to the conditions of the Creative Commons Attribution-NonCommercialNoDerivatives 4.0 International (CC BY-NC-ND 4.0) licence. Full details of this licence are available at: https://creativecommons.org/licenses/by-nc-nd/4.0/

\section{LICENCE}

CC BY-NC-ND 4.0

\section{REPOSITORY RECORD}

Yan, Liangwen, Sijung Hu, Samah Alharbi, and Panagiotis Blanos. 2019. "A Multiplexed Electronic Architecture for Opto-electronic Patch Sensor to Effectively Monitor Heart Rate and Oxygen Saturation". figshare. https://hdl.handle.net/2134/33297. 


\section{A multiplexed electronic architecture for opto-electronic patch sensor to effectively monitor heart rate and oxygen saturation}

Liangwen Yan, Sijung Hu, Samah Alharbi, Panagiotis Blanos

Liangwen Yan, Sijung Hu, Samah Alharbi, Panagiotis Blanos, "A multiplexed electronic architecture for opto-electronic patch sensor to effectively monitor heart rate and oxygen saturation," Proc. SPIE 10501, Optical Diagnostics and Sensing XVIII: Toward Point-of-Care Diagnostics, 1050115 (20 February 2018); doi: 10.1117/12.2287833

SPIE. Event: SPIE BiOS, 2018, San Francisco, California, United States 


\title{
A Multiplexed Electronic Architecture for Optoelectronic Patch Sensor to Effectively Monitor Heart Rate and Oxygen Saturation
}

\author{
Liangwen Yan ${ }^{\mathrm{a}, \mathrm{b}}$, Sijung $\mathrm{Hu}^{* \mathrm{~b}}$, Samah Alharbi ${ }^{\mathrm{b}}$ and Panagiotis Blanos ${ }^{\mathrm{b}}$ \\ ${ }^{a}$ School of Mechatronic Engineering and Automation, Shanghai University, Shanghai 200072, \\ China; lw_yan@staff.shu.edu.cn \\ ${ }^{\mathrm{b}}$ School of Mechanical, Electrical and Manufacturing Engineering, Loughborough University, Ashby \\ Road, Loughborough, Leicestershire LE1 1 3TU, UK; S.M.Alharbi@lboro.ac.uk (S.A.); \\ P.Blanos@lboro.ac.uk (P.B.) \\ *Correspondence: S.Hu@lboro.ac.uk; Tel.: +44-150-922-7058
}

\begin{abstract}
To effectively capture human vital signs, a multi-wavelength optoelectronic patch sensor (MOEPS), together with a schematic architecture of electronics, was developed to overcome the drawbacks of present photoplethysmographic (PPG) sensors. To obtain a better performance of in vivo physiological measurement, the optimal illuminations, i.e., light emitting diodes (LEDs) in the MOEPS, whose wavelength is automatically adjusted to each specific subject, were selected to capture better PPG signals. A multiplexed electronic architecture has been well established to properly drive the MOEPS and effectively capture pulsatile waveforms at rest. The protocol was designed to investigate its performance with the participation of 11 healthy subjects aged between 18 and 30 . The signals obtained from green $(525 \mathrm{~nm})$ and orange $(595 \mathrm{~nm})$ illuminations were used to extract heart rate $(\mathrm{HR})$ and oxygen saturation $\left(\mathrm{SpO}_{2} \%\right)$. These results were compared with data, simultaneously acquired, from a commercial ECG and a pulse oximeter. Considering the difficulty for current devices to attain the $\mathrm{SpO}_{2} \%$, a new computing method, to obtain the value of $\mathrm{SpO}_{2} \%$, is proposed depended on the green and orange wavelength illuminations. The values of $\mathrm{SpO}_{2} \%$ between the MOEPS and the commercial Pulse Oximeter devics showed that the results were in good agreement. The values of HR showed close correlation between commercial devices and the MOEPS (HR: $r_{1}=0.994$ (Green); $r_{2}=0.992$ (Orange); $r_{3}=0.975$ (Red); $r_{4}=0.990$ (IR)).
\end{abstract}

Keywords: Multi-wavelength Opto-electronic patch sensor (MOEPS), Oxygen saturation $\left(\mathrm{SpO}_{2} \%\right)$, Heart rate (HR)

\section{INTRODUCTION}

The photoplethysmographic (PPG) sensor technology is widely used in commercially available medical devices for measuring oxygen saturation $\left(\mathrm{SpO}_{2} \%\right)$, heart rate $(\mathrm{HR})$, and assessing autonomic function also for detecting peripheral vascular disease ${ }^{1}$. ZenPPG with two red LEDs and two infrared LEDs was developed to monitor the tissue blood perfusion ${ }^{2}$. Several $\mathrm{SpO}_{2} \%$ probes with a red LED and an infrared LED were connected to a single FPGA chip and processed in parallel so that measurement accuracy was improved ${ }^{3}$. The ring sensor was developed and it was capable of reliably monitoring a patient's heart rate and oxygen saturation $\left(\mathrm{SpO}_{2} \%\right)^{4}$. Real time analysis of $\mathrm{PPG}$ signal for $\mathrm{SpO}_{2} \%$ and pulse rate measurement was executed ${ }^{5}$. Some pulse oximeters were obtained from forehead and $\operatorname{ear}^{6 \sim 8}$. $\mathrm{The}^{\mathrm{SpO}} \mathrm{O}_{2} \%$ values from the above-mentioned sensors were calculated through the red LEDs and infrared LEDs. The camera based $\mathrm{SpO}_{2} \%$ imaging technology was applied by non-contact multiple wavelength imaging photoplethysmography ${ }^{9}$, and measurement of oxygen saturation was executed by using pulse oximeter based on fuzzy logic techique ${ }^{10}$. However, the results are not accurate enough compared with the golden standard devices. Due to the fact that skin absorption rates are usually various when the skin is illuminated by different wavelength illuminations, different results of $\mathrm{HR}$ and $\mathrm{SpO}_{2} \%$ will be obtained from different wavelength illuminations. Figure 1 illustrates an optical window of human tissue in which the illumination is greatly absorbed by melanin within the ultraviolet band $(10 \sim 400 \mathrm{~nm})$, whereas the water substance is strongly absorbed in a range of longer wavelength. Generally, the optical window $(405 \sim 1064 \mathrm{~nm})$ is often selected for opto-physiological measurements ${ }^{11}$, such as the typical application of pulse oximeter. 


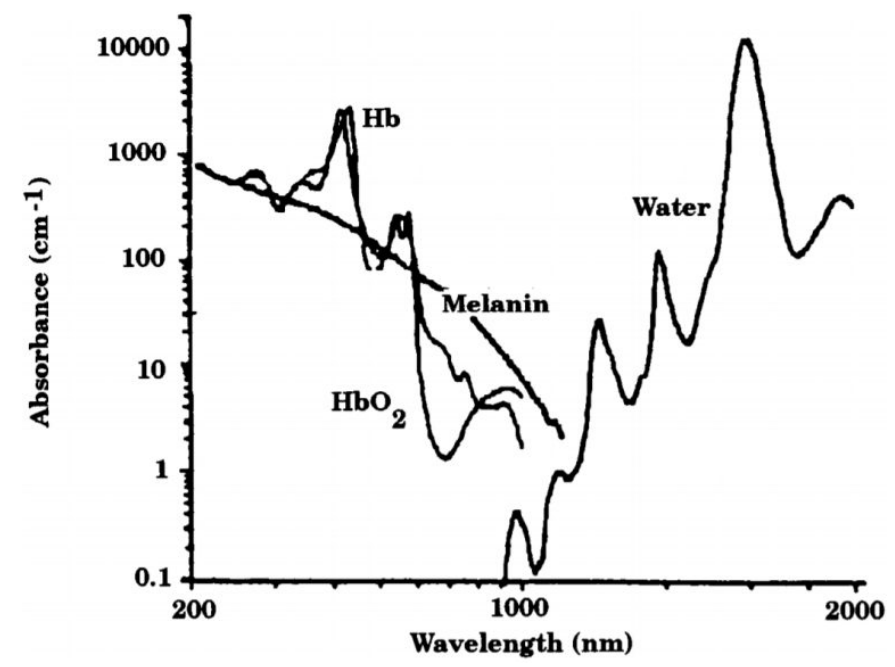

Figure 1. Skin tissue spectral window ${ }^{12}$.

In addition, the illumination wavelength of PPG is usually determined based on the absorption of oxyhemoglobin (Hb) and deoxyhemoglobin $\left(\mathrm{HbO}_{2}\right)$ associated with blood volume changes in peripheral and capillary arterial vessels. Namely, the illumination associated with specific wavelengths to skin with rich peripheral blood vessels are preferable to surface skin layers where there is no arterial blood or scattering. Table 1 shows the wavelengths of optical radiation reaching a certain penetration depth.

Table 1. Approximate penetration depth of optical radiation in skin tissue ${ }^{13}$

\begin{tabular}{|c|c|c|c|c|c|c|}
\hline Wavelength $(\mathrm{nm})$ & 500 & 600 & 700 & 800 & 1000 & 1200 \\
\hline Depth $(\mu \mathrm{m})$ & 230 & 550 & 750 & 1200 & 1600 & 2200 \\
\hline
\end{tabular}

Different optical radiation wavelengths can reach different penetration depths of skin tissue, and the illumination wavelength can be selected in the larger wavelength range, thus the $\mathrm{HR}$ and $\mathrm{SpO}_{2} \%$ can be obtained through green wavelength illumination as well as orange wavelength illumination. This study aims to calculate the HRs using different multi-wavelength illumination based on the MOEPS, and to calculate $\mathrm{SpO}_{2} \%$ through green LED and orange LED illumination. The results are compared with the golden standard devices.

\section{MULTIPLEXED AND DE-MULTIPLEXED ELECTRONIC ARCHITECTURE AND EXPERIMENTAL METHODS}

\subsection{Multiplexed and De-multiplexed Electronic Architecture}

The electronic architecture of the MOEPS system is shown in Figure 2, it consists of multiplexed and de-multiplexed circuits. The multiplexed circuit is controlled by the dsPIC microcontroller. The dsPIC microcontroller multiplexes a sequenced and synchronized illumination of 16 LED illumination sources, with four different wavelengths $(525 \mathrm{~nm}$, $590 \mathrm{~nm}, 650 \mathrm{~nm}, 870 \mathrm{~nm}$ ) using time interrupted routines and eight general purpose input/output pins (GPIO) to drive the eight input selection pins (A, B) of a four channel multiplexer. The illumination of the 16 LEDs is controlled by three different voltage levels (V1, V2, V3) through a 12-bit digital to analogue converter (DAC) via the dsPIC Microcontroller through Serial Peripheral Interface (SPI) protocol communication. The dsPIC Microcontroller enables a transistor circuit with high/low control signal to select an optimum illuminating intensity of the selective LEDs.

The de-multiplexed circuit is also controlled by the dsPIC microcontroller. A single photodiode and two identical preamplifiers are utilized to sense the light intensity after being modulated within the tissues. The detected signals are then de-multiplexed by separating the signals that represent illumination from different wavelengths and measuring the ambient light. The ambient light is then cancelled out through a differential amplifier and four raw PPG signals are acquired according to each illuminating wavelength. Four separated signals are then received by the dsPIC Microcontroller on a 10-bit analogue to digital converter (ADC) to sample them in a suitable range in order to be ready 
for processing and transmission. Finally, processed data and raw signal can be transferred through a universal asynchronous receiver/transmitter (UART) to the dsPIC Microcontroller by using a Bluetooth module.

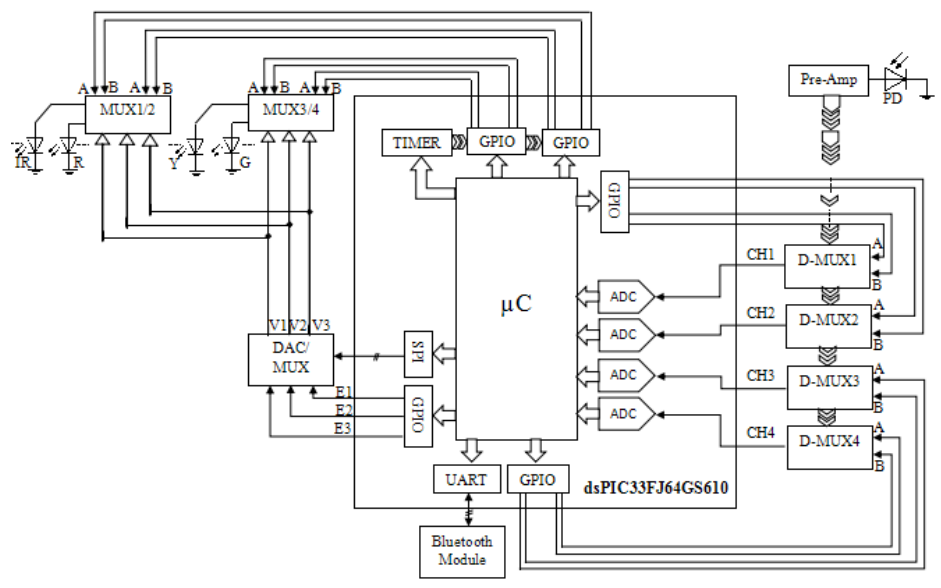

Figure 2. Electronic architecture of the multiwavelength opto-electronic patch sensor (MOEPS). The system of the MOEPS comprises of (1) MCU: microcontroller (dsPIC33FJ64GS610); (2) LED Driver (DAC/MUX); (3) MUX1/2 and MUX3/4 (Multiplexers); (4) Pre-Amp: pre-amplifiers; (5) D-MUX (De-Multiplexers); (6) ADC: analogue to digital converter; (7) Bluetooth Module

\subsection{MOEPS Configuration}

The MOEPS (physical size: $18 \mathrm{~mm} \times 18 \mathrm{~mm} \times 0.1 \mathrm{~mm}$ ), as shown in Figure 3, consists of (1) 16 light-emitting diodes (LEDs, JMSIENNA Co., Ltd., TouFen City, Taiwan) as illumination sources[14]. Their peak wavelengths were $525 \mathrm{~nm}$ (green), $590 \mathrm{~nm}$ (Orange), $650 \mathrm{~nm}$ (red) and $870 \mathrm{~nm}$ (IR) respectively, and (2) A Si-photodiode (PD) with a large active area (1.69 mm2, S10625, Hamamatsu photonics K. K., Hamamatsu City, Japan) as a photodetector. The PD and LEDs were mounted side-by-side (reflection mode PPG). The PCB routing and footprints were allocated by PADS (Pads PCB PADS Standard, Wilsonville, OR, USA). A layer of clear epoxy medical adhesive was also used to protect the optical components.

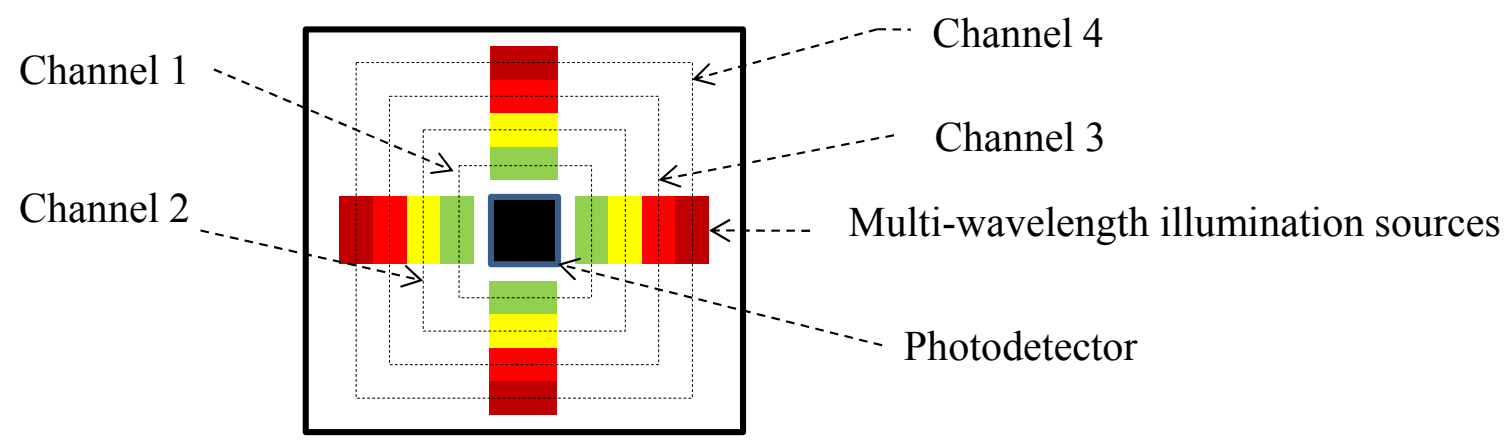

Figure 3. Schematic diagram of multiwavelength opto-electronic patch sensor (MOEPS). The multi-wavelength illumination sources of MOEPS comprises of (1) channel 1 with four green light-emitting diodes (LEDs); (2) channel 2 with four orange LEDs; (3) channel 3 with four red LEDs and (4) channel 4 with four IR LEDs.

\subsection{Electronic Composition}

The MOEPS system based on a microcontroller (dsPIC33FJ64GS610, Microchip Technology Inc., USA) implemented multi-functions control and interfacing roles. Figure 4 shows a block diagram of the MOEPS, which alters LED light intensity through voltage control. Moreover, pre-amplifiers (Pre-Amp), differential amplifier (DA) and low pass filter (LPF) were also integrated in the system device. The raw sign from the PD needs to be amplified several times by the Pre-amp. The output of the DA was transferred to a low pass filter (LPF) to eliminate high frequency such as power 
supply frequency. At that point the signal entered the processor and was converted to digital via an analogue to digital convert (ADC). The data from the MCU is sent to a PC and is then processed and separated into AC and DC signals by LabView. Referring to the AC amplitude, the LED intensities are auto-adjusted accordingly to the strength of the signal received so that high quality signals are acquired.

Since the alternative component $(\mathrm{AC})$ is $2-5 \%$ of the static component $(\mathrm{DC})^{15}$, the raw signal, acquired from the PD, needs to be amplified by the Pre-Amp. The multiplexed and the demultiplexed signals should be separated. MUX is also used as a demultiplexer to extract pulse waveform from each channel of illuminations, as the output of the PD is a pulse train of multi-wavelength illuminations. The common mode rejection of the differential amplifier (DA) is driven to a LPF to eliminate high frequency (HF), such as power supply frequency and electromagnetic. In addition, the LPF is playing an important role in preventing the aliasing components from being sampled. The signal from the LPF is then transferred to the DsPIC processor, to be later converted to a digital form through an analogue-to-digital convert (ADC). Wireless transmission is achieved by a Bluetooth module that receives the digital PPG signals from the central processor, through the USART protocol, to send them eventually to a PC.

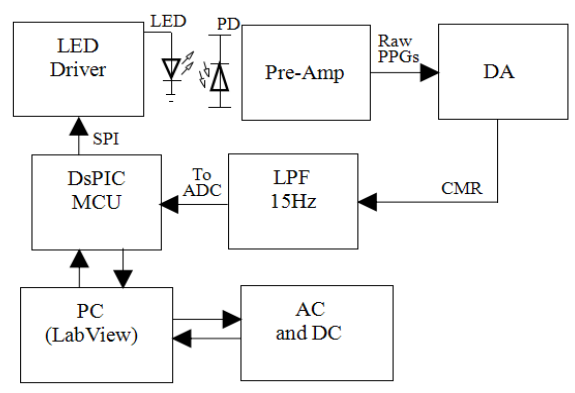

Figure 4. Block diagram of the MOEPS circuit with six individual functional modules; (1) DsPIC MCU: microcontroller (dsPIC33FJ64GS610); (2) LED Driver; (3) Pre-Amp: pre-amplifiers; (4) DA: differential amplifier; (5) LPF: low pass filter ( $\mathrm{fc}=15 \mathrm{~Hz}$ ); (6) PC with LabView software; (7) AC and DC are the separated signals from the raw data.

\subsection{Execution of the MOEPS System}

Three main functionalities operating the MOEPS system are outlined as follows:

(1) Time sequence of multiplexing LED and demultiplexing signals from the PD.

A time multiplexing algorithm is employed in the DsPIC to asynchronously switch the LEDs to "ON" and "OFF". The four channel illuminations (green, orange, red and infrared LEDs) are multiplexed at the frequency of $1 \mathrm{kHz}$. Figure 5 is a schematic diagram of time switching for multiplexed illumination of four LED channels and demultiplexing of the signals from the photodiode. The time sequence is implementing a timer interrupt for accurate performance. DsPIC is used to switch the LEDs, alternating them between two states ("OFF", "ON") providing only a single wavelength LED illumination at a time.

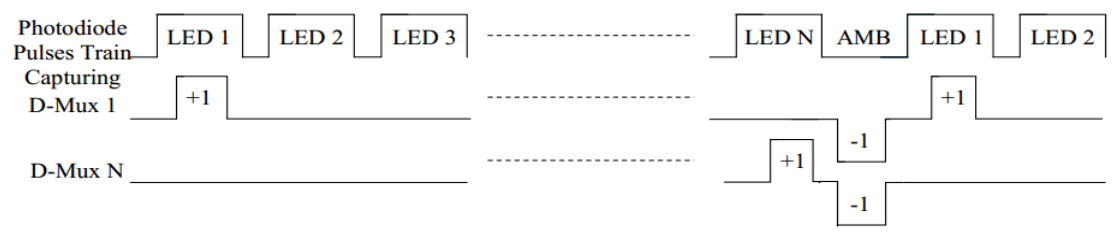

Figure 5. Schematic diagram of time switching in multiplexed illumination of LEDs and demultiplexed signals from a photodiode.

On the other hand, the switching time between these illumination sources is crucial to distinguish between different LED wavelengths, otherwise the PD produces only interference pulse trains with a combination of multi-wavelength illuminations. The output of the PD requires demultiplexing where a specific wavelength illumination is extracted from 
these combined signals at a certain time. After cancelling the effect of ambient light and common mode rejection, the signals from these individual LED illuminations, are acquired respectively and passed on to a LPF $(\mathrm{fc}=15 \mathrm{~Hz})$ where they are converted to digital signals inside the DsPIC processor.

\subsection{Experimental Protocol}

An experimental protocol with the approval of the Loughborough University Ethics Committee to examine the MOEPS system was implemented with 11 male subjects between the ages of 18 and 30 participated in the experimental protocol The subjects must be without alcohol, coffee, tea, energy drink or any drinks that affect the performance of the body for 12 hours prior to the study visit. In this protocol, the $\mathrm{HR}$ and $\mathrm{SpO}_{2} \%$ obtained from green and orange wavelength illumination by the MOEPS will be compared with the gold standard devices in rest. Prior to recording, each subject's body mass index and blood pressure were documented, as well as room temperature and humidity. The subjects were asked to perform this experiment within a 180s duration of resting, having the MOEPS attached to their palm. The HR readings were obtained from a three-lead ECG (AT-10 Plus, Schiller UK Ltd., Bellshill, UK) and the $\mathrm{SpO}_{2} \%$ readings were obtained from a commercial Pulse Oximeter (TempIRTM, China). The HR readings of the MOEPS are extracted from the raw data by the means of MATLAB (MathWorks Inc., Natick, MA, USA) based on adaptive filter signal processing. The raw data from the MOEPS can be extracted to deliver various physiological parameters such as $\mathrm{SpO}_{2} \%$. The HR measurement is based on raw data that can be determined through systolic and diastolic phases of the heart in the AC component. Using two sequence maximum peaks or two minimum troughs of PPG signal are required to measure the HR. The algorithm of HR calculation is based on the time difference $(\Delta t)$ between the detection of two successive peaks or absolute maximums. The time difference is defined as the duration of two sequenced peaks; thus the following formula is applied to measure the heart rate (HR).

$$
\mathrm{HR}(\mathrm{bpm})=(60 * \mathrm{Fs}) / \Delta \mathrm{t}
$$

Where HR is the heart rate, Fs is the sampling frequency $(256 \mathrm{~Hz})$ of PPG, 60 indicates beat per minute and $\Delta t=t_{2}-t_{1}$ is the time difference between two peaks.

Regarding the Oxygen saturation $\left(\mathrm{SpO}_{2} \%\right)$, the raw data is required to extract $\mathrm{SpO}_{2} \%$ and that can be done by using the ratio of $\mathrm{AC}$ to $\mathrm{DC}$ for both green and orange wavelength illumination.

$$
\mathrm{R}(\text { ratio of ratio })=(\text { ACGreen } / \text { DCGreen }) /(\text { ACOrange/DCOrange })
$$

The oxygen saturation $\left(\mathrm{S}_{\mathrm{P}} \mathrm{O}_{2} \%\right)$ is calculated by the following equation:

$$
\mathrm{S}_{\mathrm{P}} \mathrm{O}_{2} \%=110-25 * \mathrm{R}
$$

\section{RESULTS}

The evaluation of the HR readings between two measurement techniques, i.e., MOEPS and three-lead resting ECG were performed. Pearson's correlation analysis was also used to correlate quantitative variables, as an indicator of two techniques evolving in parallel, as shown in Figure 6. The values of HR showed close correlation between commercial devices and the MOEPS (HR: Figure 6a, $r_{1}=0.994$ (Green); Figure 6c, $r_{2}=0.992$ (Orange); Figure 6e, $r_{3}=0.975$ (Red); Figure $\left.6 \mathrm{~g}, \mathrm{r}_{4}=0.990(\mathrm{IR})\right)$.

The Bland-Altman method was used to compare the values of the HR obtained between the MOEPS and the commercial ECG devices. Figure $6 \mathrm{~b}$ indicates the bias B: 0.21 , standard deviation $\mathrm{SD}=1.96$, lower and upper limits of agreement, -

2.22 and +2.11 . Figure $6 \mathrm{~d}$ indicates the bias B: 0.0 , standard deviation $\mathrm{SD}=1.37$, lower and upper limits of agreement, 2.69 and +2.69 . Figure $6 \mathrm{f}$ indicates the bias $\mathrm{B}: 1.02$, standard deviation $\mathrm{SD}=2.36$, lower and upper limits of agreement, -3.60 and +5.64 . Figure $6 \mathrm{~h}$ indicates the bias B: 0.28 , standard deviation $\mathrm{SD}=1.80$, lower and upper limits of agreement, -2.96 and +3.68 . 


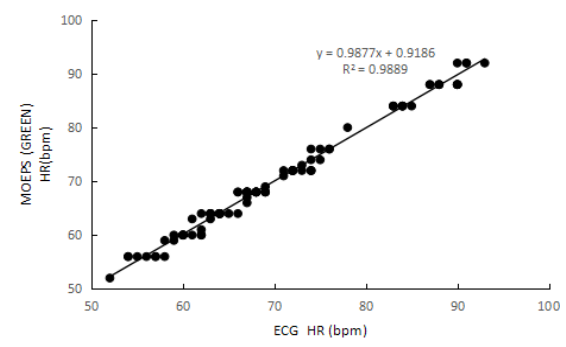

a. HR Correlation between MOEPS (GREEN) and ECG with $(r=0.994)$.

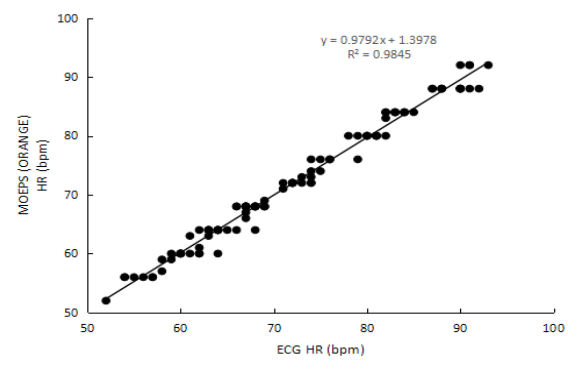

c. HR Correlation between MOEPS (ORANGE) and ECG with $(r=0.992)$.

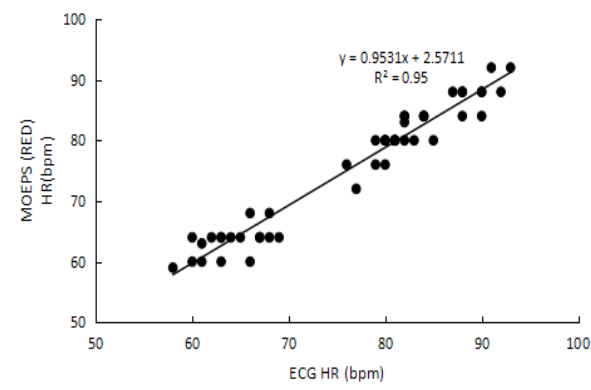

e. HR Correlation between MOEPS (RED) and ECG with $(r=0.975)$.

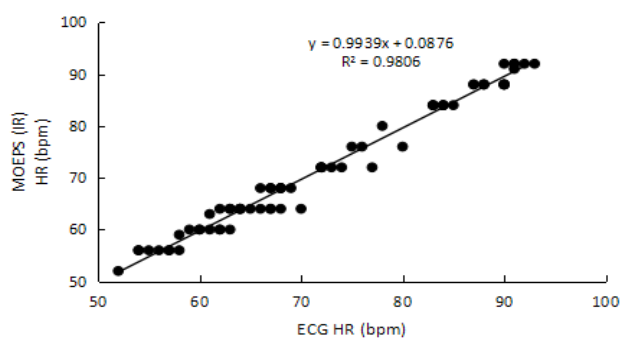

g. HR Correlation between MOEPS (IR) and ECG with

$$
(\mathrm{r}=0.990) \text {. }
$$

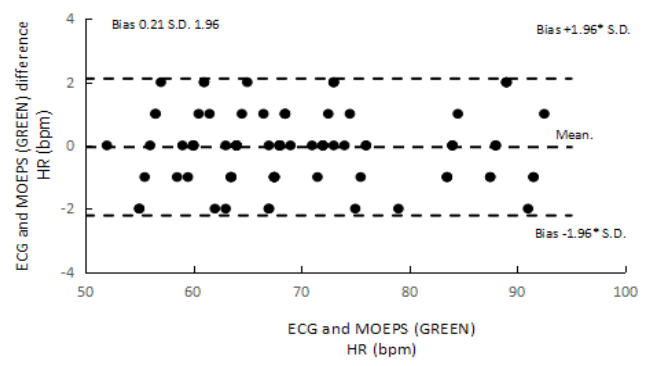

b. Differences in HR (GREEN) outputs recorded at resting using Bland-Altman plot. The acceptable range: $\mathrm{B} \pm 1.96 \mathrm{SD}$.

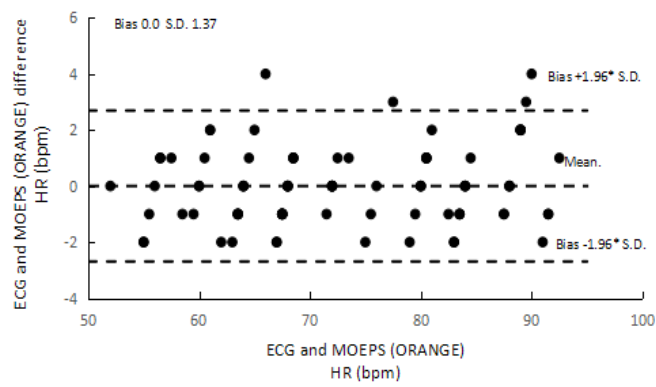

d. Differences in HR (ORANGE) outputs recorded at resting using Bland-Altman plot. The acceptable range: $\mathrm{B} \pm 1.96 \mathrm{SD}$.

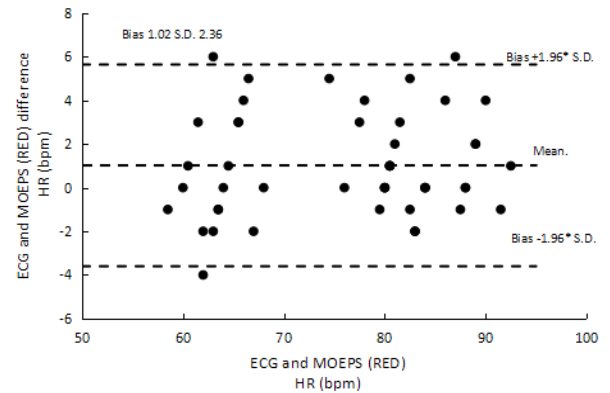

f. Differences in HR (RED) outputs recorded at resting using Bland-Altman plot. The acceptable range: $\mathrm{B} \pm 1.96 \mathrm{SD}$

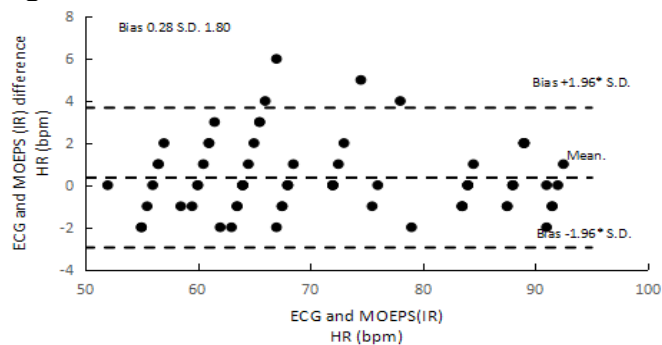

h. Differences in HR (IR) outputs recorded at resting using Bland-Altman plot. The acceptable range: $\mathrm{B} \pm 1.96 \mathrm{SD}$.

Figure 6. HR Correlation between MOEPS and ECG during rest mode; Differences in HR outputs recorded at resting using Bland-Altman plot, the acceptable range: $\mathrm{B} \pm 1.96 \mathrm{SD}$. 
The Bland-Altman method was used to compare the values of the $\mathrm{SpO}_{2} \%$ obtained between the MOEPS and the commercial Pulse Oximeter devices. Figure 7 indicates the bias B: 0.40, standard deviation $\mathrm{SD}=1.96$, lower and upper limits of agreement, -3.48 and +4.27 respectively.

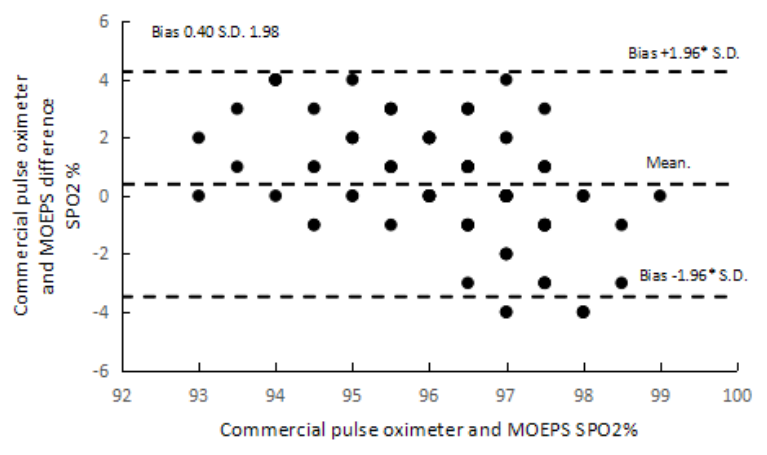

Figure 7. Differences in $\mathrm{SpO}_{2} \%$ outputs recorded at resting using Bland-Altman plot. The acceptable range: $\mathrm{B} \pm 1.96 \mathrm{SD}$.

\section{DISCUSSION}

The outcomes obtained from the implementation of the experimental protocol have elucidated the MOEPS together with its associated electronics and algorithms in the following aspects:

1) fulfill the requirements of clinical grade physiological monitoring as the comparison study between commercial three-lead resting ECG and the MOEPS was implemented.

2) The optimal illumination were be selected by a desired spectral wavelength from $525 \mathrm{~nm}$ to $870 \mathrm{~nm}$, together with four channels of 16 LEDs, through an auto-adaptive adjustment. Undoubtedly, multi-wavelength illuminations, for instance having more LEDs attached to the MOEPS, could improve the performance of opto-physiological monitoring.

3) Compared among Figure 6a, 6c, 6e and 6g, the HR (GREEN) measurement values between the MOEPS and the threelead ECG have a highest correlation (r: 0.994). As depicted in the Bland-Altman plot in Figure 6b, 6d, 6f and 6h, the HR outputs recorded at resting, are most likely to be in the acceptable range of $\mathrm{B} \pm 1.96 \mathrm{SD}$ in the HR difference between the three-lead resting ECG and the MOEPS.

4) From Figures 5, it can be derived that the HR measurements, obtained from the commercial ECG device and the MOEPS, are not only compatible but also satisfactory. Specifically, compared among Figure 6b, 6d, 6f and 6h, their lower and upper limits are -2.22 and $+2.11,-2.69$ and $+2.69,-3.60$ and $+5.64,-2.96$ and +3.68 , the Figure $6 \mathrm{~b}$ is smallest fluctuation range while Figure $6 \mathrm{f}$ is biggest fluctuation range. Thus the HRs obtained from green wavelength illumination are more accurate than those obtained from other wavelength illumination. As presented in Figure 6, the $\mathrm{SpO}_{2} \%$ were calculated by means of green illumination and orange illumination of the MOEPS. Comparing the values of $\mathrm{SpO}_{2} \%$ between the MOEPS and the commercial Pulse Oximeter devices, the bias B: 0.40, standard deviation SD = 1.96 , lower and upper limits of agreement, -3.48 and +4.27 , showed that the results were in good agreement.

\section{CONCLUSIONS}

A multi-wavelength opto-electronic patch sensor (MOEPS), through the implementation of a designated exercise protocol, has been proved to effectively detect physiological signals. The study shows the approach of acquiring and processing physiological signals that could be effectively applied in real time, such as sending signals to a PC monitor or a server on a receiving end user. With a comparison measurement, the compatibility of HRs between the ECG and the MOEPS was proven to be in an acceptable range of the HR difference using the three-lead ECG and the MOEPS. Meanwhile, the compatibility of $\mathrm{SpO}_{2} \%$ between the commercial Pulse Oximeter and the MOEPS was proven to be in an acceptable range of the $\mathrm{SpO}_{2} \%$ difference using the commercial Pulse Oximeter and the MOEPS. Specifically, the outcome of the experimental protocol, with the participation of 11 healthy male subjects, demonstrates the MOEPS 
ability to measure effectively $\mathrm{HR}$ and $\mathrm{SpO}_{2} \%$ from green wavelength illumination and orange wavelength illumination. Therefore, the devices obtaining $\mathrm{HR}$ and $\mathrm{SpO}_{2} \%$ from red wavelength illumination and infrared wavelength illumination may be replaced by those obtaining $\mathrm{HR}$ and $\mathrm{SpO}_{2} \%$ from green wavelength illumination and orange wavelength illumination. Furthermore, the MOEPS could be consolidated to already available wearable and smart devices for in-line and real-time monitoring and assessment. Nevertheless, several challenges, such as different MOEPS measurement locations on the skin with relevantly rich peripheral blood vessels, motion artifact, and even electronic noises, are to be addressed in the upcoming studies.

Acknowledgments: The authors are grateful to all the members from Loughborough University and Shanghai University for their enthusiastic support. Also, the authors would like to thank the financial support of NSFC (Grants No. 61371149) for this study.

Author Contributions: L. Yan conducted the electronic validation and prepared the manuscript. S. Alharbi carried out the signal processing. P. Blanos executed the experimental protocol. S. Hu supervised the project and guided the project implementation, and organized the manuscript.

Conflicts of Interest: The authors declare no conflict of interest.

\section{REFERENCES}

[1] Allen, J., "Photoplethysmography and its application in clinical physiological measurement," Physiol. Meas. 28, R1-R39 (2007).

[2] Abay, T.Y., Kyriacou, P. A. "Reflectance photoplethysmography as noninvasive monitoring of tissue blood perfusion," IEEE Transactions on Biomedical Engineering, 62(9), 2187-2195 (2015).

[3] Stojanovic, Radovan, Dejan, K., "Design of an oximeter based on LED-LED configuration and FPGA technology," Sensors, 13 (1), 574-586 (2013).

[4] Asada, H. H., Phillip, S., Andrew, R., Sokwoo, R., Reginald, C. H., "Mobile monitoring with wearable photoplethysmographic biosensors," IEEE engineering in medicine and biology magazine, 22(3), 28-40 (2003).

[5] Bagha, Sangeeta, and Laxmi Shaw, "A real time analysis of PPG signal for measurement of SpO2 and pulse rate," International journal of computer applications, 36(11), 45-50 (2011).

[6] Kocher, Serge, Roman Rohling, and Andres Tschupp, "Performance of a digital PCO2/SPO2 ear sensor," Journal of clinical monitoring and computing, 18 (2), 75-79 (2004).

[7] Shelley, Kirk H., et al., "The effect of venous pulsation on the forehead pulse oximeter wave form as a possible source of error in SpO2 calculation," Anesthesia \& Analgesia, 100 (3), 743-747 (2005).

[8] Nuhr, M., et al., "Forehead $\mathrm{SpO} 2$ monitoring compared to finger $\mathrm{SpO} 2$ recording in emergency transport," Anaesthesia, 59(4), 390-393 (2004).

[9] Wieringa, Fokko P., Frits Mastik, and Antonius FW van der Steen, "Contactless multiple wavelength photoplethysmographic imaging: a first step toward 'SpO2 camera' technology," Annals of biomedical engineering. 33(8), 1034-1041 (2005).

[10] Ateș, Gözde, and Kemal Polat, "Measuring of oxygen saturation using pulse oximeter based on fuzzy logic,." Medical Measurements and Applications Proceedings (MeMeA), 2012 IEEE International Symposium on. IEEE, 2012.

[11] Spigulis, J., Gailite, L., Alexey L., Renars E., "Multi-wavelength photoplethysmography for simultaneous recording of skin blood pulsations at different vascular depths," Appl. Optic, 46, 1754-59 (2007).

[12] Rebecca, R.K., Eva, S.M.,"Quantitative optical spectroscopy for tissue diagnosis," Annu. Rev. Phys. Chem. 47, 555-606 (1996).

[13] Anderson, R., Parrish, J., "The optics of human skin," J. Invest Dermatol. 77, 13-19 (1981) .

[14]Hu, S., Azorin-Peris, V., "Opto-Physiological Sensor and Method of Assembly," UK International Patent Application No. PCT/GB2014/053095, WO 2015056007 A1. (2015).

[15] Meglinski, IV., Matcher, SJ., "Computer simulation of the skin reflectance spectra," Comput. Method. Program. Biomed. 70, 179-86 (2003). 